\title{
A deeper insight into the dip coating process in the presence of insoluble surfactants: A numerical analysis
}

\author{
Diego M. Campana, ${ }^{1,2}$ Sebastián Ubal, ${ }^{1,2}$ María D. Giavedoni, ${ }^{1, a)}$ Fernando A. Saita ${ }^{1}$ \\ ${ }^{1}$ Intec (CONICET-UNL) Güemes 3450, 3000 Santa Fe, Argentina \\ ${ }^{2}$ Facultad de Ingeniería, UNER, C.C. 47 Suc. 3, 3100 Paraná, ER, Argentina
}

(Received 20 December 2010; accepted 18 April 2011; published online 23 May 2011)

\begin{abstract}
A numerical investigation is carried out to study the effects of an insoluble surfactant on the dip coating of a flat substrate. Predictions of both the film thickness and the concentration of surfactant in the film as a function of the capillary number compare well with the solutions of a simpler asymptotic model based on the lubrication approximation. Streamline patterns confirm the existence of a stagnation point located in the bulk phase in the region of the dynamic meniscus - a conjecture postulated forty years ago. The evolution of the flow patterns and the interfacial variables shows how the classical result of Landau and Levich is recovered as the coating speed is augmented. Finally, we show that the effect of inertia forces cannot be neglected when the viscosity of the coating liquid is low. (C) 2011 American Institute of Physics.
\end{abstract}

[doi:10.1063/1.3589346]

\section{INTRODUCTION}

Dip coating has probably been the most extensively studied coating flow since the pioneering work carried out by Landau and Levich. ${ }^{1}$ This process deposits a thin uniform film on a solid by withdrawing it at a constant speed from a pool of the liquid to be coated. Landau and Levich were the first to obtain an approximate solution to predict the thickness of the film formed at very small substrate speeds, i.e., when viscous and capillary forces balance each other; thus, dip coating is usually referred to as the Landau-Levich problem. Thereafter, numerous works have been performed to assess the influence on the film thickness of usually present forces that were not considered in their analysis (viz., gravity, ${ }^{2,3}$ inertia, ${ }^{4}$ Marangoni stresses due to the adsorption of surface active agents on the interface,,$^{5}$ etc.).

With regards to the presence of surfactant on the process, it must be remarked that soluble surfactants have been much more studied than insoluble ones. In most of the experimental studies published on the subject, the coating fluid is an aqueous solution of surfactant and the substrate is either a cylindrical fiber ${ }^{5,6}$ or a flat plate. ${ }^{7}$ All those works show that the film tends to thicken when a surfactant is added to the original clean system. The common explanation to the above observation is the so called Marangoni effect: the nonuniform distribution of surfactant, which is a consequence of the surface stretching, gives rise to surface tension gradients that generate a traction that pulls more liquid into the film region. The experiments of Krechetnikov and Homsy ${ }^{7}$ $(\mathrm{K} \& \mathrm{H})$ were conducted at a parameter range where the adsorption of surfactant from the bulk to the interface is fast enough to compensate the dilution produced by the stretching of the free surface and, consequently, Marangoni stresses are not likely to be responsible for the observed film thickening. In an attempt to disclose the underlying mechanisms

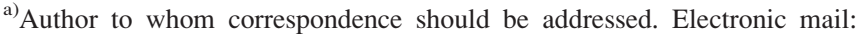
madelia@santafe-conicet.gov.ar. involved in the thickening process under the operating conditions assumed by $\mathrm{K} \& \mathrm{H}$, we carried out a numerical analysis ${ }^{8}$ to mimic their experiments; the study is based on the hydrodynamic modeling of the surfactant effect and shows predictions of the thickening factor (i.e., the ratio between the thickness of the contaminated system and the LandauLevich $^{1}$ for a uniformly distributed surfactant) in very good agreement with the experimental ones. The numerical solutions show that the elastic effects of the surfactant reduce the interfacial curvature all along the dynamic meniscus; thus, the pressure gradient (i.e. the gradient of the capillary pressure) that opposes the action of viscous forces is also reduced, and more liquid is pulled towards the film region. The changes detected in the flow field near the interface associated with the surfactant are another interesting feature of that work.

The influence of an insoluble surfactant has been studied by Park ${ }^{9}$ in the limit of very small capillary number (i.e., low coating speeds). He used the method of matched asymptotic expansions to obtain equations for the film thickness and the interfacial distribution of the surface active agent in the following two cases. One, relevant to the preparation of Langmuir Blodgett films, corresponds to relatively high concentration of surfactants with small spatial variations. The other one, typical of surface contamination, assumes that the concentration of surfactant is low but its spatial variation can be large. The results of the analysis show that the surfactant affects the film thickness of the entrained film within a range of capillary number that depends on the magnitude of the elasticity parameter (see the definition of $\beta$ in Sec. II A). In fact, within that range, the thickening factor is a nonmonotonic function of the capillary number: at the lowest end, surface diffusion is important enough to produce a uniform distribution of surfactant, while at the other end, viscous forces overcome surface elasticity. Therefore, in both limits the film thickness can be well approximated by the LandauLevich law and the thickening factor is equal to 1 . In between 
those extremes, the thickening factor first increases and then decreases as the capillary number is augmented. Park also found that the maximum value of that parameter is equal to $4^{2 / 3}$. It is worthy to note that Ratulowski and Chang ${ }^{10}$ in their analysis about the effects of surfactants on the film left behind by a large bubble displacing a liquid in a capillary tube predicted that the film could increase by the same maximum factor when the transport of surfactant in the film is limited by a bulk concentration gradient existing in that region.

Up to our knowledge, there is not an analysis of the dip coating process under the effects of an insoluble surfactant based on the solution of the full hydrodynamic problem; however, Krechetnikov and Homsy ${ }^{11}$ in their numerical work on the effects of a soluble surfactant on the LandauLevich problem argue that the presence of an insoluble surface active agent has no effect on the film thickness. Their assertion is based on numerical solutions-not presented in the paper-that show that the stagnation point lying on the interface of a clean system remains on the interface when an insoluble surfactant is adsorbed. Thus, the surfactant is swept away by convection from the interface.

The objective of the present work is two-fold. On the one hand, our goal is to corroborate the results obtained by Park; on the other hand, we aim at extending those results by considering inertia forces and gaining a better comprehension about the mechanisms by which insoluble surfactants act upon dip coating processes. For that purpose, we numerically solve the Landau-Levich problem when an insoluble surfactant is adsorbed at the interface.

We found that the trend followed by the film thickness as a function of the capillary number and the elasticity parameter of the surfactant are qualitatively similar to those reported by Park in his asymptotic analysis and thus are in contradiction with the assertion of Krechetnikov and Homsy. ${ }^{11}$ In fact, the numerical solutions reported here show that the stagnation point present at the interface of a clean system is displaced towards the bulk and thus the surfactant remains adsorbed confirming the hypothesis of Groenveld. ${ }^{12}$ We also discuss the changes experienced by the flow patterns near the meniscus and the solid wall when the coating speed is augmented up to a value at which the film thickness of the system can be approximated by the Landau-Levich law. In addition, when the values of the physical parameters used by Park are considered, we show that the influence of inertia forces is not negligible; therefore, they must be taken into account when insoluble surfactants are present in the dip coating process.

The paper is organized as follows. In Sec. II, we present the governing equations and their boundary conditions and we briefly outline the numerical technique employed to solve them. In Sec. III, we discuss the effects of an insoluble surfactant on the Landau-Levich problem, first when inertia is neglected and then when this force is considered. Finally, Sec. IV is devoted to the conclusion.

\section{MODEL FORMULATION}

\section{A. Governing equations and boundary conditions}

We consider the two dimensional flow produced when a solid plate moves out of a large liquid bath at a constant

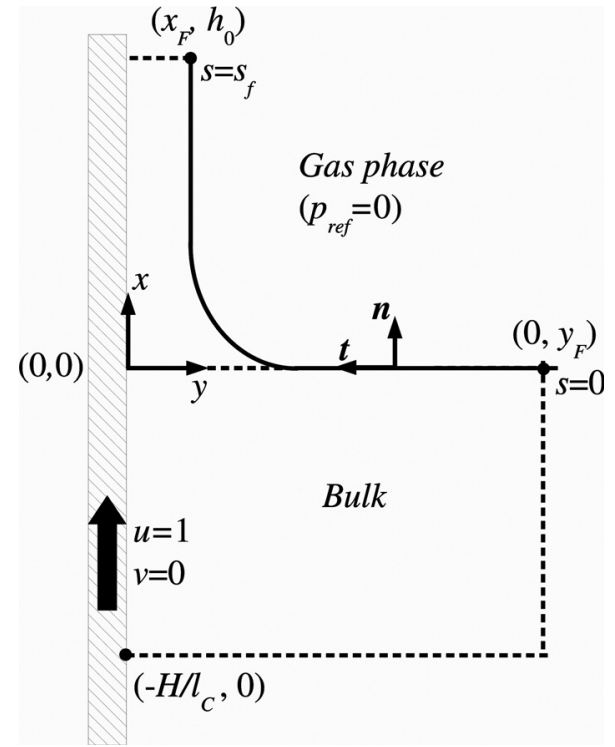

FIG. 1. Sketch of the flow domain and coordinate system adopted.

speed, $U$. The viscosity, $\mu$, and density, $\rho$, of the coating liquid are constant and the air above is regarded as inviscid, its pressure is constant and set equal to zero. In Figure 1, a schematic representation of the problem and the system coordinate adopted are illustrated. There is an insoluble surfactant adsorbed along the free surface whose concentration is kept constant and equal to $\Gamma_{R E F}$ at $x=0, y=y_{F}$. Therefore, the flow is governed by continuity and Navier-Stokes equations which in the coordinate system adopted are

$$
\begin{gathered}
u_{x}+v_{y}=0 \\
\operatorname{Re}\left(u u_{x}+v u_{y}\right)=-\frac{1}{C a} p_{x}+u_{x x}+u_{y y}-\frac{1}{C a}, \\
\operatorname{Re}\left(u v_{x}+v v_{y}\right)=-\frac{1}{C a} p_{y}+v_{x x}+v_{y y} .
\end{gathered}
$$

In the above expressions, subscripts denote partial derivatives and the variables are written in dimensionless form using the following scales: $U$ for velocities, $l_{C}=\left(\sigma_{R E F} / \rho g\right)^{1 / 2}$ for lengths, and $\sigma_{R E F} / l_{C}$ for pressures; $R e=\rho U l_{C} / \mu$ is the Reynolds number and $C a=\mu U / \sigma_{R E F}$ is the capillary number; and $\sigma_{R E F}$ is the surface tension at $\left(0, y_{F}\right)$. Boundary conditions are as follows:

(a) The fluid adheres to the moving plate, then $u=1$ and $v=0$ at $y=0$.

(b) At the film exit boundary which is located far away from the meniscus $\left(x=x_{F}, 0 \leq y \leq h_{0}\right)$, the flow becomes unidirectional and the film thickness is uniform and equal to $h_{0}$; thus, we impose $u_{x}=v_{x}=0$ and $u_{y}=1 / C a\left(y-h_{0}\right)$ as Neumann boundary conditions.

(c) At the bottom of the domain $\left(x=-H / l_{C}, y \geq 0\right)$, the flow does not change along the $x$-coordinate; therefore, $u_{x}$ and $v_{x}$ are set equal to zero. Also, a uniform pressure is weakly imposed along this boundary. This pressure is equal to the pressure at the lowest right corner of the domain and is obtained by introducing an additional equation in the numerical code that sets the slope of the free 
surface equal to zero at $\left(0, y_{F}\right)$. The difference between the calculated and the hydrostatic pressure does not exceed $0.01 \%$. (See Ref. 8 for more details).

(d) At the lateral boundary of the domain which is located far away from the moving plate $\left(-H / l_{C} \leq x \leq 0, y=y_{F}\right)$, the surface becomes flat and the flow does not change along the $y$-coordinate; thus, $u_{y}$ and $v_{y}$ are set equal to zero.

The free surface is a material surface; therefore, the usual form of the kinematic condition applies, that is,

$$
\mathbf{v} \cdot \mathbf{n}=0
$$

where $\mathbf{n}$ being the unit normal vector to the free surface pointing toward the gas phase. When there are surfactants in the system and the interface is regarded as Newtonian, the coefficients of the surface stress tensor (i.e., surface viscosities and surface tension) depend on the local concentration of surfactant. In this work, we assume that both surface viscosity coefficients are negligible; thus, the traction vector along the interface results as follows:

$$
-\frac{1}{C a} p \mathbf{n}+\mathbf{n} \cdot\left[\nabla \mathbf{v}+(\nabla \mathbf{v})^{T}\right]=\frac{1}{C a} \frac{d(\sigma \mathbf{t})}{d s},
$$

where $s$ is the arc length along the interface measured from $\left(0, y_{F}\right)$ to $\left(x_{F}, h_{0}\right), \mathbf{t}$ is the unit tangent vector to the free surface pointing in the direction of increasing $s$, and $\sigma$ is the local value of the surface tension measured in units of $\sigma_{R E F}$ and depends on the concentration of surfactant. Following Park, we assume a linear relationship between the surface tension and the interfacial concentration of surfactant

$$
\sigma=1-\beta(\Gamma-1)
$$

In the above expression, $\Gamma$ is dimensionless concentration of surfactant measured in units of $\Gamma_{R E F}$ and $\beta=-\frac{\Gamma_{R E F}}{\sigma_{R E F}}\left(\frac{\partial \sigma}{\partial \Gamma}\right)_{\Gamma_{R E F}}$ is the elasticity number.

The transport equation for the surfactant on the free surface is ${ }^{13}$

$$
\nabla_{S} \cdot\left(\Gamma \mathbf{v}^{S}\right)-\frac{1}{P e_{S}} \nabla_{S}^{2} \Gamma=j_{n}
$$

where $\mathbf{v}^{S}$ is the surface velocity, $P e_{S}=U l_{C} / D_{S}$ is the surface Péclet number, $D_{S}$ being the surface diffusion coefficient of the surfactant, and $j_{n}$ is the flux of surfactant between the bulk and the free surface. In the present case, $j_{n}=0$ because the surfactant is insoluble.

Finally, the following boundary conditions complete the formulation of the problem

$$
\Gamma=1, \quad \frac{d y^{F S}}{d s}=0 \quad \text { at } s=0, \text { and } \frac{d x^{F S}}{d s}=\frac{d \Gamma}{d s}=0 \text { as } s=s_{f}
$$

In the above expression, $x^{F S}$ and $y^{F S}$ stand for the $x$ and $y$ coordinates of the free surface, respectively. In addition, $s=0$ is located at a distance $y_{F}$ from the moving substrate; this distance is large enough to assume that the free surface is already flat. Also, at this location the concentration of surfactant is fixed. At first glance, this last condition might seem arbitrary; however, it represents the only feasible "steady state" experiment in a finite bath. In fact, if the concentration of solute is not fixed, its value will continuously diminish due to the convective transport of surfactant towards the film region and no steady state will be achieved. Equation (8) also establishes that far away from $x=0$ (in the thin film region), the free surface is parallel to the solid surface.

\section{B. Solution method}

A description of the numerical scheme used to solve Eqs. (1)-(8) can be found in Ref. 8. Therefore, in this Section, we will discuss the tests carried out to establish the appropriateness of the domain extension as well as the number of the elements and their distribution in the computational grid.

The location of the bottom boundary must guarantee that the flow normal to the plane $x=-H / l_{C}$ does not appreciably changes with $x$, and the position of the upper boundary must assure the fulfillment of the conditions there imposed to the flow as well as to the interfacial concentration of surfactant and the interface. Since those are the same criteria adopted when the effects of a soluble surfactant were studied, ${ }^{8}$ similar numerical tests were carried out in the present case. From those tests, $H / l_{C}=-80$ and $x_{F}=30$ proved to be adequate for the computations discussed in this work.

In the hypothetical case of a perfect insoluble surfactant and when the mass transport is dominated by convection, the solution is sensitive to the location of the $y_{F}$ coordinate; in fact, the inflow of surfactant to the system depends on its concentration and on the surface velocity at $s=0$. In our formulation, the former variable is fixed and the latter depends on the distance to the solid wall. The criterion adopted in this work to establish the extension of the flow domain in the $y$-direction is that the magnitude of the derivative of the surfactant concentration at $s=0$ be smaller than $2 \times 10^{-3}$ for the largest capillary number used in the simulations when $R e=0$. Tests carried out show that if $y_{F}$ is equal to 100 , the above requirement is satisfied. It is worth to note that the largest value of $|d \Gamma / d s|_{s=0}$ detected in the computations does not exceed 0.005 when $m=\operatorname{Re} / \mathrm{Ca}=52500$ and $\beta=0.01$ or 0.1 (see Fig. 2).

To select a finite element mesh, we looked for the invariance of the solution with the number of grid elements. In particular, we require that the film thickness be independent of further refinements with a tolerance smaller than $1 \%$. The three meshes tested, whose main features are indicated in Table I, satisfied that criterion even in the worse conditions, i.e., large Reynolds number and small elasticity parameter. Moreover, in most of the numerical tests carried out, changes in $h_{0}$ did not exceed $0.1 \%$ when results corresponding to the three meshes were compared. In view of the above facts and considering the computational cost, all numerical solutions reported in this work were computed with $M 2$. 


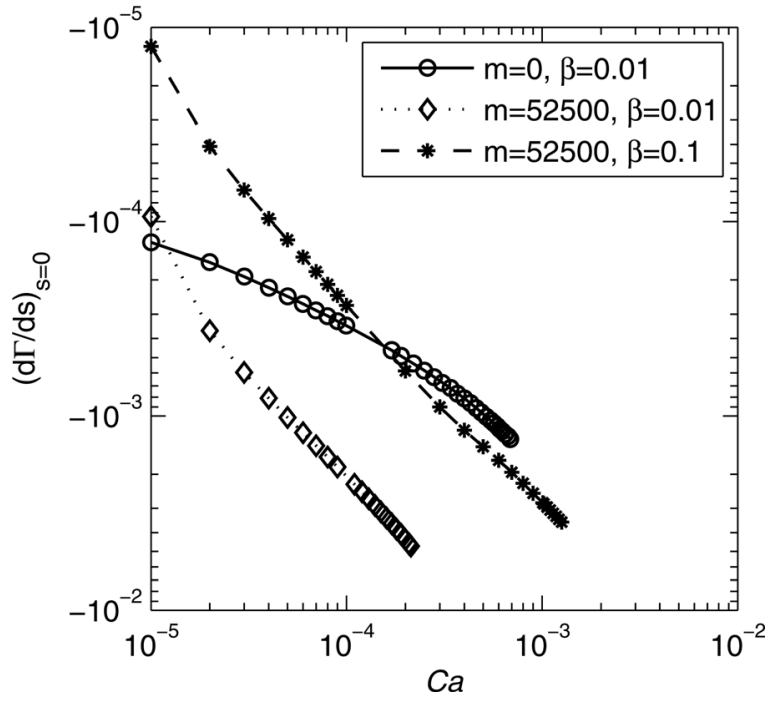

FIG. 2. $(d \Gamma / d s)_{s=0}$ as a function of the capillary number for $m=52500$ and $\beta=0.01$ and 0.1 when $y_{F}=100$.

\section{RESULTS AND DISCUSSION}

The most relevant result of a dip coating study is the prediction of the film thickness of the coated layer as a function of the physical parameters of the process, particularly its dependence on the substrate speed.

To study the effect produced by an insoluble surfactant on the thickness of the coated layer, we firstly define the system to be analyzed by fixing the physical properties of both the liquid and the surfactant. Once the governing equations are solved for a certain substrate speed, the latter is incremented by a convenient amount and the dimensionless numbers that depend on $U$ are recalculated accordingly.

We use values proposed by Park for the physical parameters of the system, that is, liquid viscosity and liquid density are set equal to $10^{-3} \mathrm{~Pa} \mathrm{~s}$ and $10^{3} \mathrm{~kg} \mathrm{~m}^{-3}$, respectively; the reference surface tension is $0.030 \mathrm{~N} \mathrm{~m}^{-1}$ and the surface diffusion coefficient is $10^{-9} \mathrm{~m}^{2} \mathrm{~s}^{-1}$. For the selected values of the physical variables and within the range of capillary number (i.e., coating speeds) considered, the surface Péclet number varies between 525 and $5.25 \times 10^{5}$; therefore, the interfacial distribution of surfactant largely depends on the free surface velocity. Also, under these conditions, the parameter $m$ defined as the ratio between inertia and surface forces (i.e., $m=R e / C a=\rho \sigma_{R E F} l_{C} / \mu^{2}$ ) is equal to 52500, and consequently, the Reynolds number is negligible only for very small $\mathrm{Ca}$. In order to compare our solutions with Park's, we first discuss the predictions computed by setting $R e=0$ in the numerical code and then we assess the influ-

TABLE I. Main features of the three meshes tested. $E$ is the number of elements, $N_{\text {total }}$ and $\mathrm{N}_{F S}$ are the number of nodes in the domain and the free surface, respectively, and $N_{D F}$ is the number of unknowns.

\begin{tabular}{lcrrc}
\hline \hline Mesh & $E$ & $N_{\text {total }}$ & $N_{F S}$ & $N_{D F}$ \\
\hline M2 & 18200 & 74482 & 721 & 167646 \\
M3 & 30000 & 121241 & 1001 & 273501 \\
M4 & 36000 & 145241 & 961 & 327501 \\
\hline \hline
\end{tabular}

ence of inertia by repeating the calculations with the Reynolds number corresponding to each selected $\mathrm{Ca}$. The analysis of the results for $R e=0$ is divided into two parts; in the first, we study the problem when elastic forces are not negligible, whereas in the second, we consider the behavior of the system when those forces are null, i.e., when the film thicknesses of the contaminated system can be predicted by the Landau-Levich law.

\section{A. $R e=0$}

\section{Non-negligible elastic effects}

Numerical predictions for the film thickness as a function of the Capillary number for $\beta=0.01$ and 0.1 are depicted in Fig. 3. Solutions of the asymptotic model when $\beta=0.01$ and $\beta=0.1$ taken from Figure 5 of Park's paper are included for comparison. From those curves the following features are apparent:

(i) The presence of the surfactant gives rise to a thicker film within a range of $\mathrm{Ca}$ that depends on the elasticity number. The range becomes broader as $\beta$ is increased.

(ii) For any value of $\beta$, the thickness of the film in the contaminated system can be at most equal to $4^{2 / 3}$ the thickness of the corresponding clean system (dotted line in Fig. 3).

(iii) As $\mathrm{Ca}$ increases, that is, as the ratio between viscous and surface forces becomes larger, the film thickness approaches the Landau-Levich law (dashed line in Fig. 3).

It is evident that the same trend is followed by the solution of the approximate model; however, a closer comparison shows that the numerical film thicknesses are below the asymptotic ones and that the differences become larger when both the capillary and the elasticity number are augmented. Park in his analysis neglects the effect of gravity and imposes that the liquid attains the solid speed in the flat film region; therefore, it is likely that the differences between both predictions are due to this approximation. This

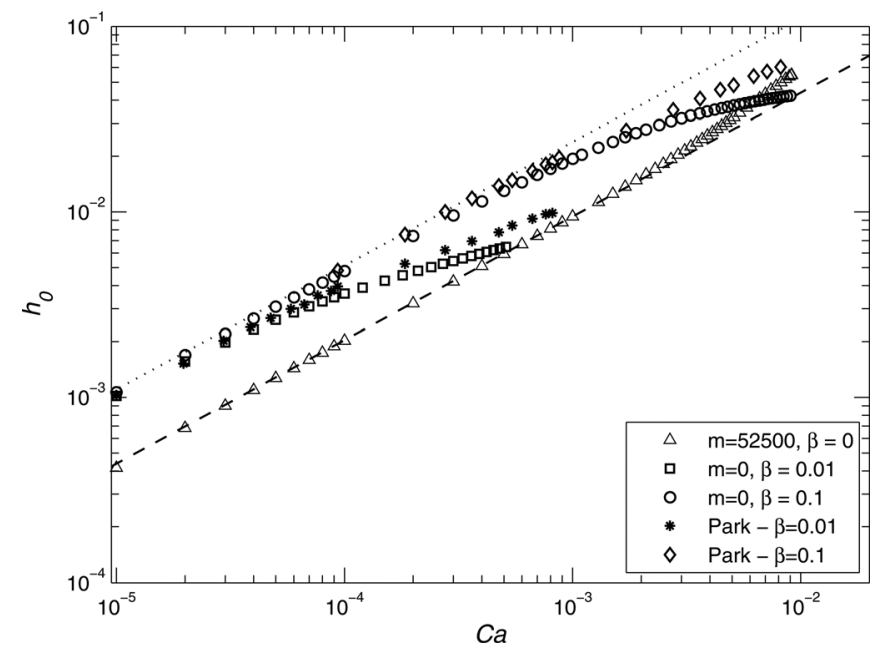

FIG. 3. Dimensionless film thickness as a function of $\mathrm{Ca}$ for $\beta=0.01$ and 0.1 . 
speculation is supported by the fact that discrepancies increase as the gravity effects become more important, i.e., for thicker films deposited at larger values of $C a$ and $\beta$.

The numerical algorithm fails to converge when the solutions of the contaminated system and the Landau-Levich predictions merge into one curve. A close inspection of the interfacial variables, in particular of the surface concentration of surfactant, shows that at that point this variable goes to zero in the region where the dynamic meniscus meets the film and thus, the lack of convergence could result from a deficient mesh refinement. This issue will be addressed more extensively in Sec. III A 2.

Typical flow patterns of the Landau-Levich problem in the absence of surface active agents show the presence of a stagnation point at the interface in the dynamic meniscus, more precisely, where the film thickness is approximately three times the final film thickness $\left(h_{0}\right)$. According to results depicted in Fig. 3 showing film thickening, that point cannot be present when an insoluble surfactant is adsorbed along the interface; otherwise, as long as surface diffusion is negligible, the surface active solute will be convected towards the ends of the free surface and, consequently, it will not act upon the dip coating process. This issue that is discussed by Park and is suggested in a previous work in which we studied the effects of a soluble surfactant ${ }^{8}$ will be addressed in this paper when analyzing the flow fields. Thus, the interfacial concentration of surfactant diminishes from $s=0$ toward the film and this gradient gives rise to Marangoni tractions $\left(T_{n s}=-\frac{\beta}{C a} \frac{d \Gamma}{d s}\right)$ which in turn pull more liquid into the film region. Those tractions increase with $\beta$ and diminish with $\mathrm{Ca}$; thus, as $U$ is augmented for any value of $\beta$ viscous forces first equilibrate and then exceed surface forces reducing the thickening effect until the thickness of the corresponding clean system is finally attained.

In a previous paper, ${ }^{8}$ we showed that the thickening factors larger than one detected when the coating liquid contains a soluble surfactant are mainly associated with changes in both the interfacial shape and the pressure distribution in the dynamic meniscus and not with the nonzero values detected in the tangential component of the surface stress.

Typically, in the static meniscus region, the liquid pressure decreases linearly along the $x$-coordinate (see Fig. 1) until it matches the dynamic meniscus and then it rapidly increases with $x$ to reach the air pressure and, consequently, the pressure gradient presents a maximum. Results reported in Ref. 8 point out that the Marangoni forces stretch the transition region and reduce the surface curvature, leading to a smaller adverse pressure gradient along the dynamic meniscus; thus, more liquid is pulled into the film region for a given $\mathrm{Ca}$ when the elasticity number is augmented.

To establish whether or not the same mechanism contributes to the film thickening observed in the present analysis, we evaluated the maximum value of $d p / d x$ for the same set of parameters as in Fig. 3; results are illustrated in Fig. 4. We see that also in this case, the presence of the surface active agent reduces the pressure gradient detected in the corresponding clean system, larger differences are detected at lower values of the capillary number where curves for $\beta=0.1$ and 0.01 approach each other in correspondence with

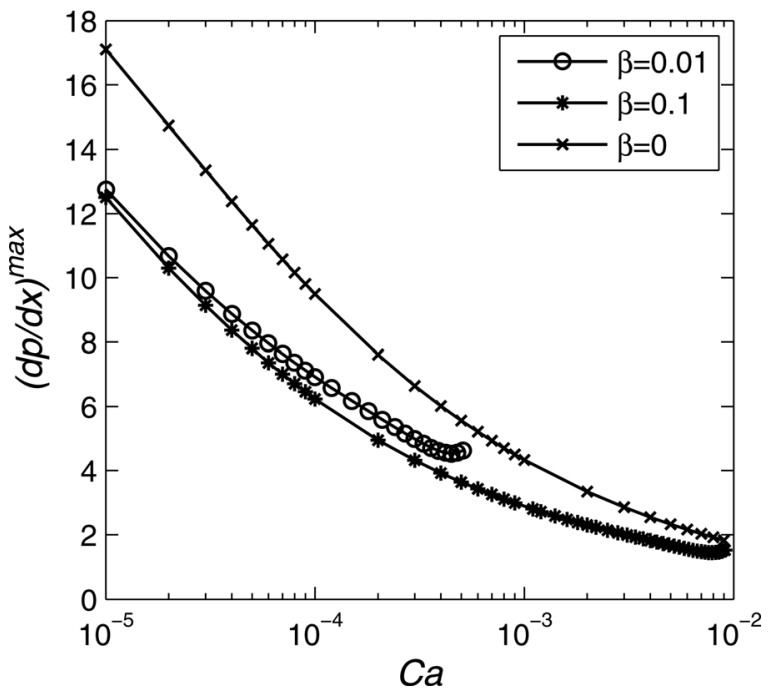

FIG. 4. Maximum pressure gradient as a function of $C a$ for $\beta=0.01,0.1$, and $0 ; m=0$.

results depicted in Fig. 3 for the film thickness. Numerical solutions (not reported here) point out that the length of the dynamic meniscus is stretched by the surfactant; for instance, it is approximately equal to twice the length of the corresponding clean system when $C a=0.00127$ and $\beta=0.1$. Then, thickening factors larger than one resulting from the adsorption of an insoluble surface active agent are consequence of both a nonvanishing tangential surface stress and a change in the free surface shape which in turns modifies the pressure distribution.

Other variable of interest is the concentration of surfactant in the film, this is, particularly true when the surfactant is an additive which confers specific properties to the coated layer. In Fig. $5, \Gamma_{s f}=\Gamma\left(s=s_{f}\right)$ is depicted as a function of $\mathrm{Ca}$ for $\beta=0.01$ and 0.1 ; values computed with Eq. (58) $(\beta=0.1)$ or picked from Fig. $6(\beta=0.01)$ of Ref. 9 are also drawn for comparison. According to Park, that the expression provides a good approximation to the solution of the asymptotic model when $\beta=0.1$ and $C a \leq 10^{-3}$.

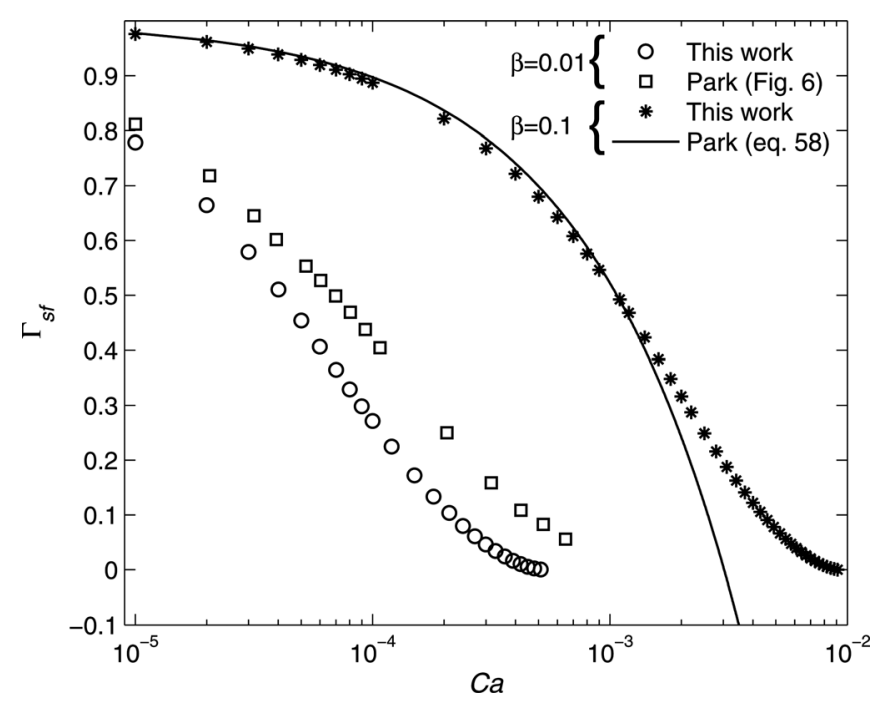

FIG. 5. Interfacial concentration of surfactant in the film region as a function of the capillary number for $\beta=0.01$ and 0.1 . 


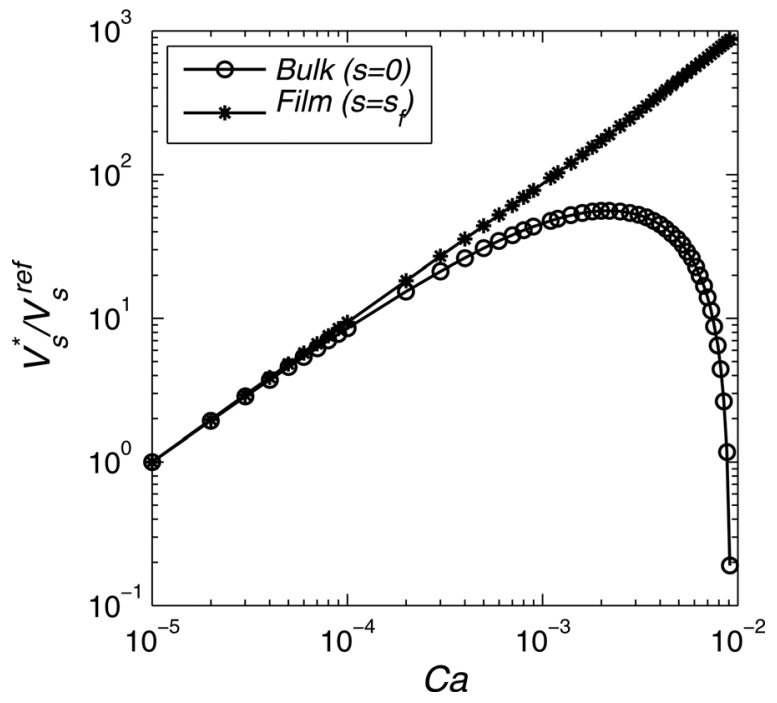

FIG. 6. Interfacial dimensional velocities at both ends of the free surface measured in units of the corresponding velocities for $C a=1 \times 10^{-5}$ and $\beta=0.1$.

It is easy to see that numerical and asymptotic predictions follow the same trend: the concentration diminishes as $\mathrm{Ca}$ is augmented and it becomes nearly equal to zero when the Landau-Levich law is approached. It is remarkable the good correspondence observed between the solutions of the full hydrodynamic problem and those calculated with Eq. (58) when $C a \leq 10^{-3}$ and $\beta=0.1$. If results illustrated in Figs. 3 and 5 are analyzed together, it is concluded that the best agreement between the simplified and the full models takes place in the region where elastic effects prevail over viscous effects. Seemingly, when those forces compete, differences between both solutions become more noticeable; finally, if viscous force rules the behavior of the system, the lubrication approximation and the 2-D model will draw close to the Landau-Levich law.

Since the characteristic velocity is the substrate speed, to put into evidence the influence of this variable on the free surface velocity, and in turn on the convective transport of solute, we evaluate the ratio between the magnitude of the dimensional surface velocity at both ends of the interface for a given $\mathrm{Ca}$ (denoted as $V_{S}^{*}$ ) and the corresponding ones to the same value of $\beta$ and $C a=10^{-5}$ (denoted as $V_{S}^{\text {ref }}$ ); the results for $\beta=0.1$ are illustrated in Fig. 6. It is worthy to note that the analogous curves for $\beta=0.01$ (not shown here) are qualitatively similar.

Figure 6 indicates that the dimensional interfacial velocity in the film region $\left(s=s_{f}\right)$ increases almost linearly (as expected) with $\mathrm{Ca}$, i.e., almost in the same proportion as the substrate speed is augmented. On the other hand, the dimensional velocity at $s=0$ increases until it reaches a maximum at $C a \approx 2.2 \times 10^{-3}$ and thence continuously diminishes to become negligible near the value of $\mathrm{Ca}$ at which the LandauLevich expression provides a good approximation for the film thickness. In other words, as the film increases its velocity with the substrate speed, the liquid located on the surface of the pool and far away from the substrate $(s=0)$ presents a nonmonotonic behavior characterized by a quasistagnant condition at higher capillary values. This behavior strongly affects

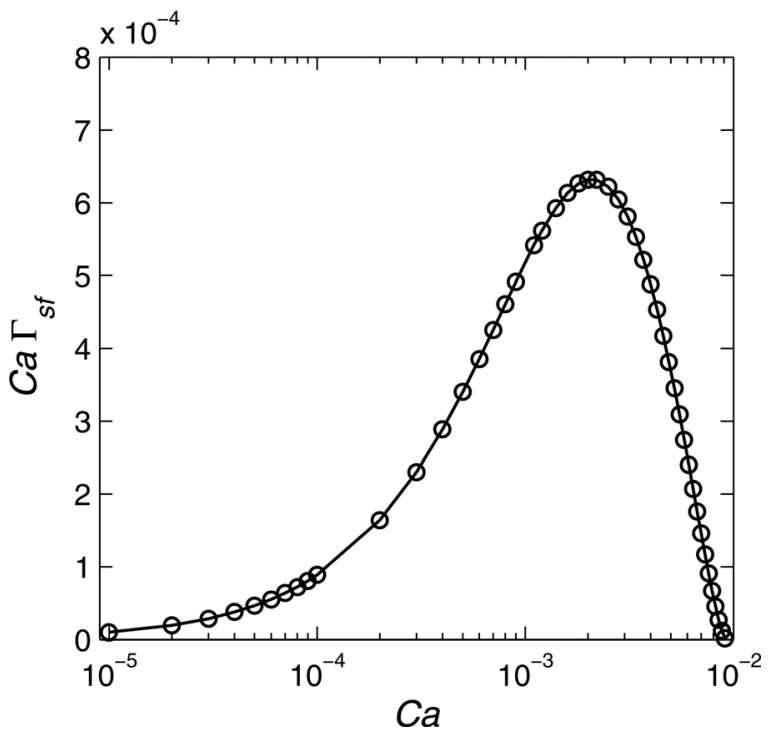

FIG. 7. $\left(C a \times \Gamma_{s f}\right)$ vs. $C a$ for $\beta=0.1$.

the interfacial transport of surfactant; in fact, if interfacial diffusion is neglected, the rate of supply of solute to the film exclusively depends on the velocity at $s=0$ where the concentration of solute is kept constant. Therefore, since the inflow and outflow rates must be equal, previous results point out that the amount of surfactant per unit time carried out by the liquid film will attain a maximum when $C a \approx 2.2 \times 10^{-3}$ and will be negligible for $C a \approx 10^{-2}$. This feature can be observed in Fig. 7 where the quantity $\left(C a \times \Gamma_{s f}\right)$, which is a measure of the mass outflow rate of surfactant, is drawn as a function of $C a$.

Results depicted in Fig. 6 show that the difference between both velocities is almost zero at low values of $\mathrm{Ca}$ but it continuously increases with this parameter at a rate that is also increasing particularly for values of $\mathrm{Ca}$ larger than 0.0022 . Therefore, one might expect larger concentration gradients as this parameter is augmented.

The interfacial velocity and the distribution of surfactant along the free surface for $\beta=0.1$ and four values of the capillary number illustrated in Figs. $8(\mathrm{a})$ and $8(\mathrm{~b})$ are in
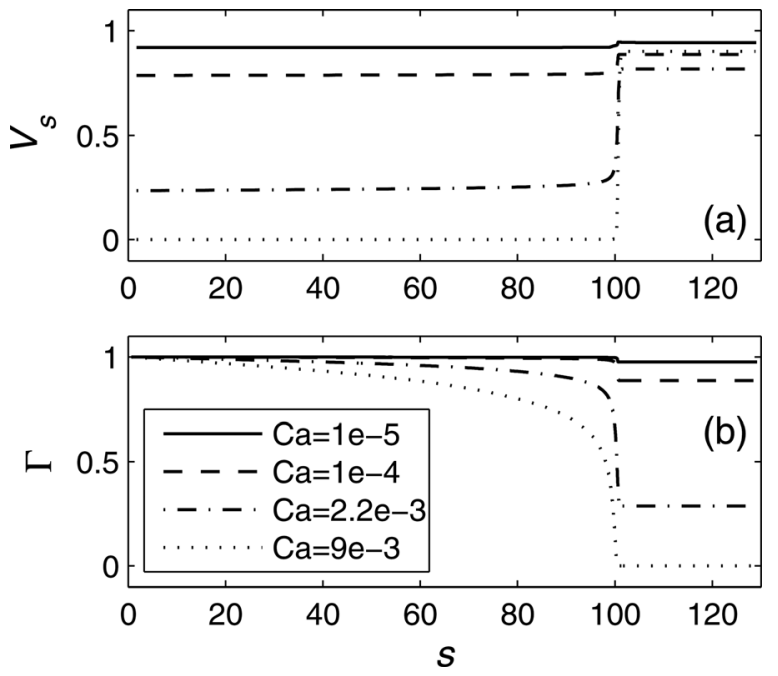

FIG. 8. Interfacial velocity (a) and distribution of surfactant along the free surface (b) for $\beta=0.1$. 

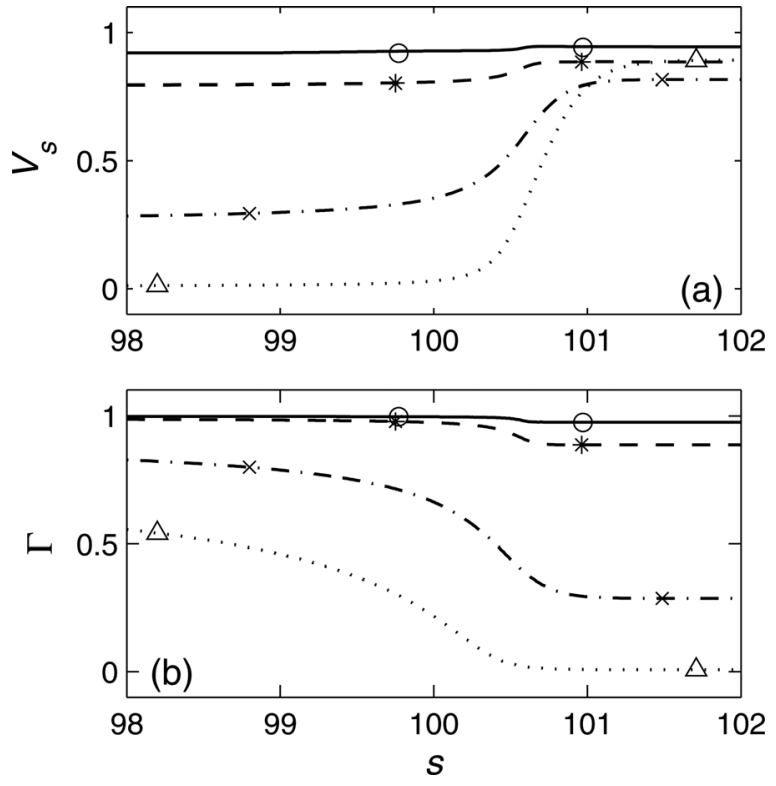

FIG. 9. Free surface velocity (a) and interfacial concentration of surfactant (b) along the dynamic meniscus when $\beta=0.1$. The lines correspond to the same values of $\mathrm{Ca}$ as in Fig. 8, while symbols on each curve represent the approximate location where the dynamic meniscus joins either the static or the film region.

agreement with the above speculations. In fact, for $C a=10^{-5}$, the velocity is nearly constant along the free surface and so is the concentration of surfactant which in the film region is approximately equal to $98 \%$ of the reference concentration. A similar behavior is detected for $C a=10^{-4}$, although now the concentration of surfactant in the film and at $s=0$ differs by nearly $10 \%$. A closer inspection of those curves (see Figs. 9(a) and 9(b)) reveals that the maximum of $|d \Gamma / d s|$ always occurs at the dynamic meniscus. In addition, the maximum of $|d \Gamma / d s|$ is a nonmonotonic function of the capillary number; the largest maximum is detected for $C a \approx$ $2.2 \times 10^{-3}$ (see Fig. 9(b)) .

If we now look at the curve for $C a=0.0022$, we see that the difference in the concentration of surfactant at both ends of the interface is more noticeable: $\Gamma\left(s_{f}\right) / \Gamma(0) \approx 0.3$. In agreement with this result, the ratio between the dimensional surface velocity at $s=s_{f}$ and at $s=0$ is approximately equal to 3.3 (see Fig. 6). The most remarkable changes between the distribution of surfactant for $C a=0.0022$ and those corresponding to the lowest values of this parameter considered in Fig. 8 are the strong reduction in the amount of surfactant adsorbed in the film region and the slight diminution detected along the static meniscus; consequently, for $C a=0.0022$, there is a large concentration gradient in the dynamic meniscus (see Fig. 9(b)).

If the coating speed is further increased, the concentration of surfactant in the film is drastically reduced (see the curve for $C a=0.009$ in Fig. 8(b)) in agreement with the striking variation experienced by $V_{S}^{*}\left(s_{f}\right) / V_{S}^{*}(0)$ (Fig. 6); actually, for $C a=0.009, V_{S}^{*}\left(s_{f}\right) / V_{S}^{*}(0) \approx 4000$ and $\Gamma\left(s_{f}\right) / \Gamma(0) \approx 0.00025$. Also, as the concentration of surfactant in the film diminishes, a smoother distribution is observed along the dynamic meniscus resulting from the larger gradient that exists in the static region (see the curve for $C a=0.009$ in Fig. 9(b)).

An interesting conclusion that can be drawn from Figs. 6 and $8(a)$ is that the flow pattern must change significantly near the free surface as the coating speed is augmented. To analyze this point, we calculated the stream function and drew selected streamlines near the interface and the solid wall for four different values of the capillary number; they are illustrated in Fig. 10. It should be noticed that the flow patterns shown pertain to the dynamic meniscus region which approximately extends from $s=99.5$ to $s=101.0$. At low $C a$, i.e., when the interfacial concentration of surfactant is quite uniform, there is a stagnation point in the bulk (marked with a dot in the figure) very close to the solid wall-less than $0.01 l_{C}$ away from it. The film is mainly formed by two streams, one is close to the substrate and the other one is flowing all along the interface. These two streams give rise to the two inverted $\mathrm{V}$-shape regions shown in Fig. 10(a) which converge at the stagnation point.

At first when $U$ is progressively augmented, the stagnation point moves downward away from the wall and the interface (see Fig. 10(b)) and the amount of liquid dragged by the substrate and the free surface towards the film region increases; thus, a thicker coating layer is formed. The analysis of a sequence of flow patterns (not shown here) suggests that the downward motion of the stagnation point is observed within the range of $C a$ where $V_{S}^{*}(0)$ increases with the coating speed.

When $C a$ is larger than approximately 0.0022 , an increment of this parameter (i.e., of the substrate speed) is accompanied by the displacement of the stagnation point towards the interface and away from the substrate. The size of the $\mathrm{V}$-shape region located in the vicinity of the free surface is largely reduced and so is the amount of liquid dragged by the interface toward the film (see Fig. 10(c)). When the film thickness approaches the Landau-Levich law, the amount of liquid flowing parallel to the free surface towards the film region is so small that the liquid in its vicinity is almost stagnant. The length of this low velocity region increases with the capillary number and ends at a stagnation point located close to the interface (black dot in Fig. 10(d)). It is likely that more than one stagnation point exists; nevertheless, that region is so narrow that we were not able to obtain a more accurate picture of the flow field in there.

The resulting flow pattern resembles that of a clean system, even though in the contaminated case, the surface velocity does not change sign (it is approximately equal to 0.0005 and to 0.02 at $s=0$ and in the neighborhood of the bulk stagnation point represented with a black dot in Fig. $10(d)$, respectively).

\section{The Landau-Levich limit}

When discussing the above results, we conjectured that the lack of convergence of the algorithm when the system approaches the Landau-Levich law could be due to an insufficient mesh refinement. That hypothesis is based on the evolution of the interfacial variables particularly in the trend followed by the concentration of surfactant as that limiting 

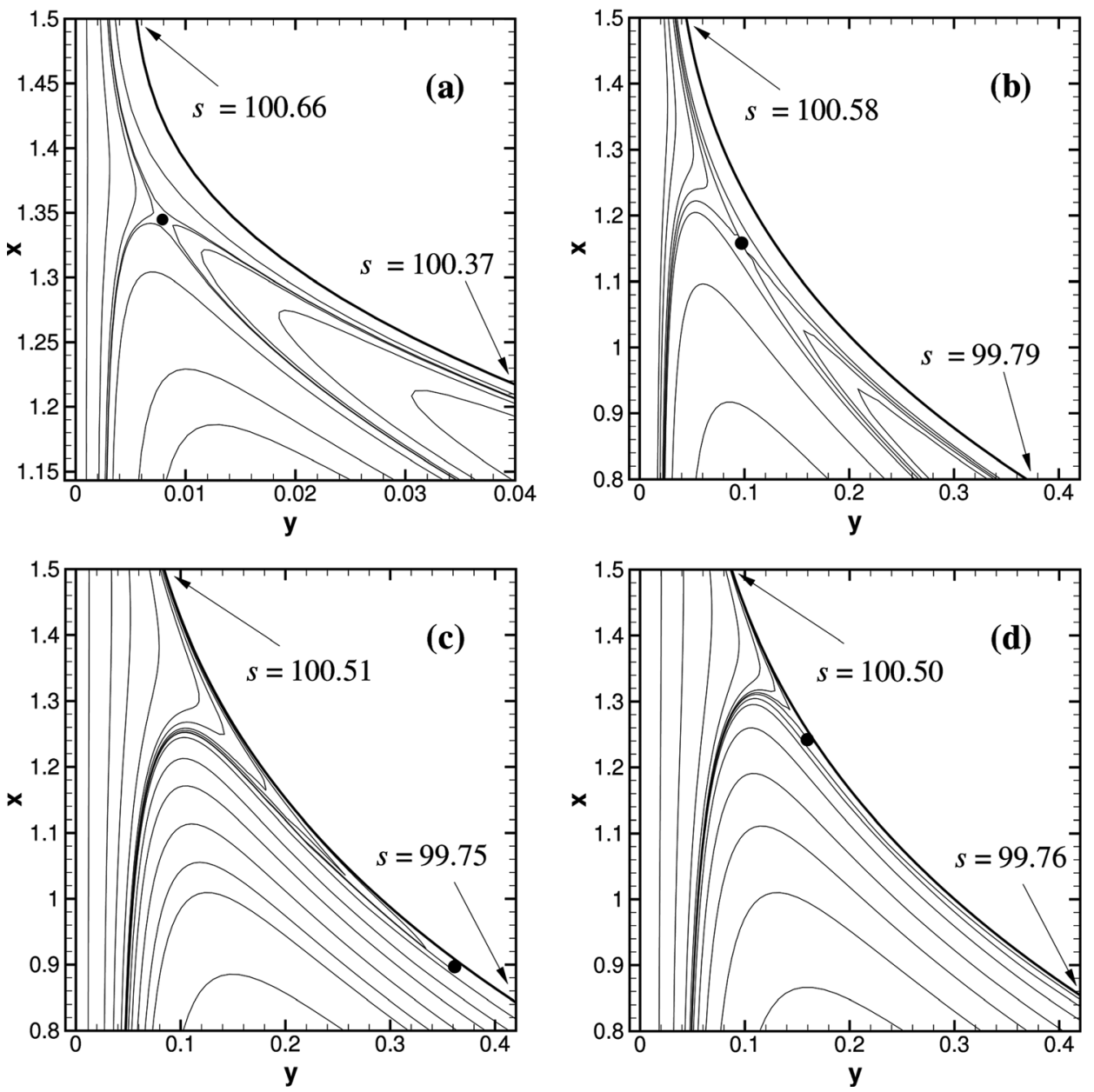

FIG. 10. Selected streamlines near the solid wall and the interface for $\beta=0.1$, $C a=1 \times 10^{-4}$ (a); $\beta=0.1, C a=0.0022$ (b); $\beta=0.1, C a=0.008$ (c); and $\beta=0.1$, $C a=0.009$ (d). The matching between the static and the dynamic menisci takes place at $s$ approximately equal to 99.75 (a), 98.79 (b), 98.39 (c), and 98.20 (d). behavior is reached. In fact, curves portrayed in Figs. 7, 8(b), and 9(b) show that the film is depleted of surfactant for the particular set of $C a$ and $\beta$ at which the film thicknesses of the contaminated and clean systems are nearly equal. Also, at this point, results illustrated in Fig. 8(a) show that a large interfacial region is nearly stagnant. Thus, to follow the evolution of the system in a range of the parameters where viscous forces wipe out the effects of the surfactant, a very fine mesh in the zone where the concentration of surfactant becomes zero might be required.

To prove that this is indeed the case, we carried out the following numerical test. Instead of using a finer mesh, we computed solutions for $\beta=0.001$, i.e., a value of the elasticity number at which the transition takes place at smaller substrate speed. The distribution of surfactant along the interface is governed by Eq. (7) with $j_{n}=0$, when the solute concentration approaches zero, the term $\nabla_{S} \Gamma \approx 0$. Therefore, if $P e_{S}$ is very large, the convective nature of the process will be strongly reinforced. In correspondence with that observation, the computed interfacial distribution of the surface active agent presents oscillations when the capillary number is slightly augmented beyond the limits considered in Fig. 3 for $\beta=0.01$ and 0.1 , i.e., immediately before the algorithm fails to converge. Two common ways to avoid those unphysical oscillations are to use either a very fine grid or a stabilizing scheme such as upwinding or PetrovGalerkin methods.
When $\beta=0.001$, the transition to the Landau-Levich regime takes place at $C a \approx 2.5 \times 10^{-5}$ and $P e_{S} \approx 1837$, that is, the surface Péclet number is approximately 14 times smaller than the largest value considered in the computations carried out for $\beta=0.01$; thus, one should expect that diffusion will be large enough to eliminate (or at least largely reduce) the oscillations. Results reported in Fig. 11 support our

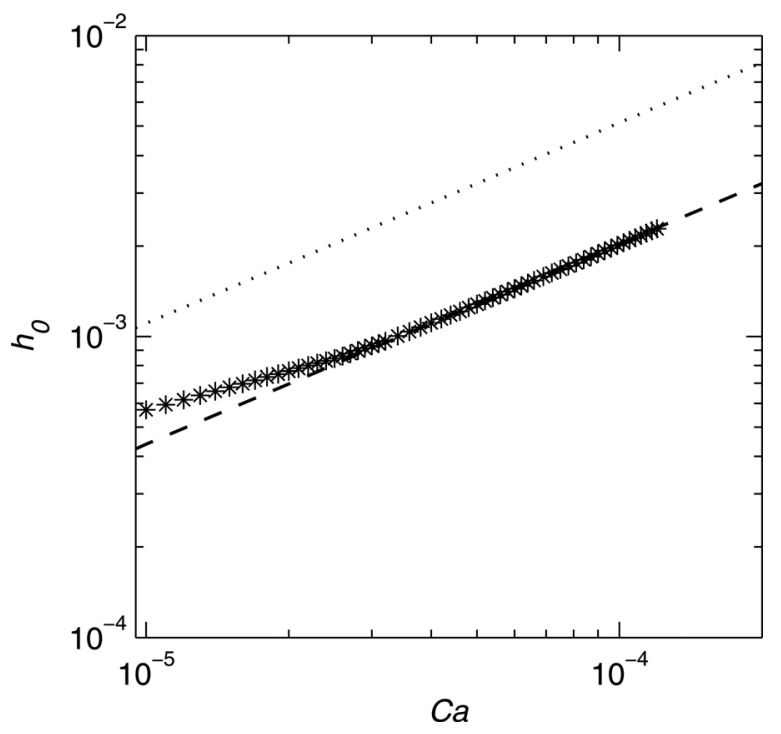

FIG. 11. Dimensionless film thickness as a function of $C a$ for $\beta=0.001$. 

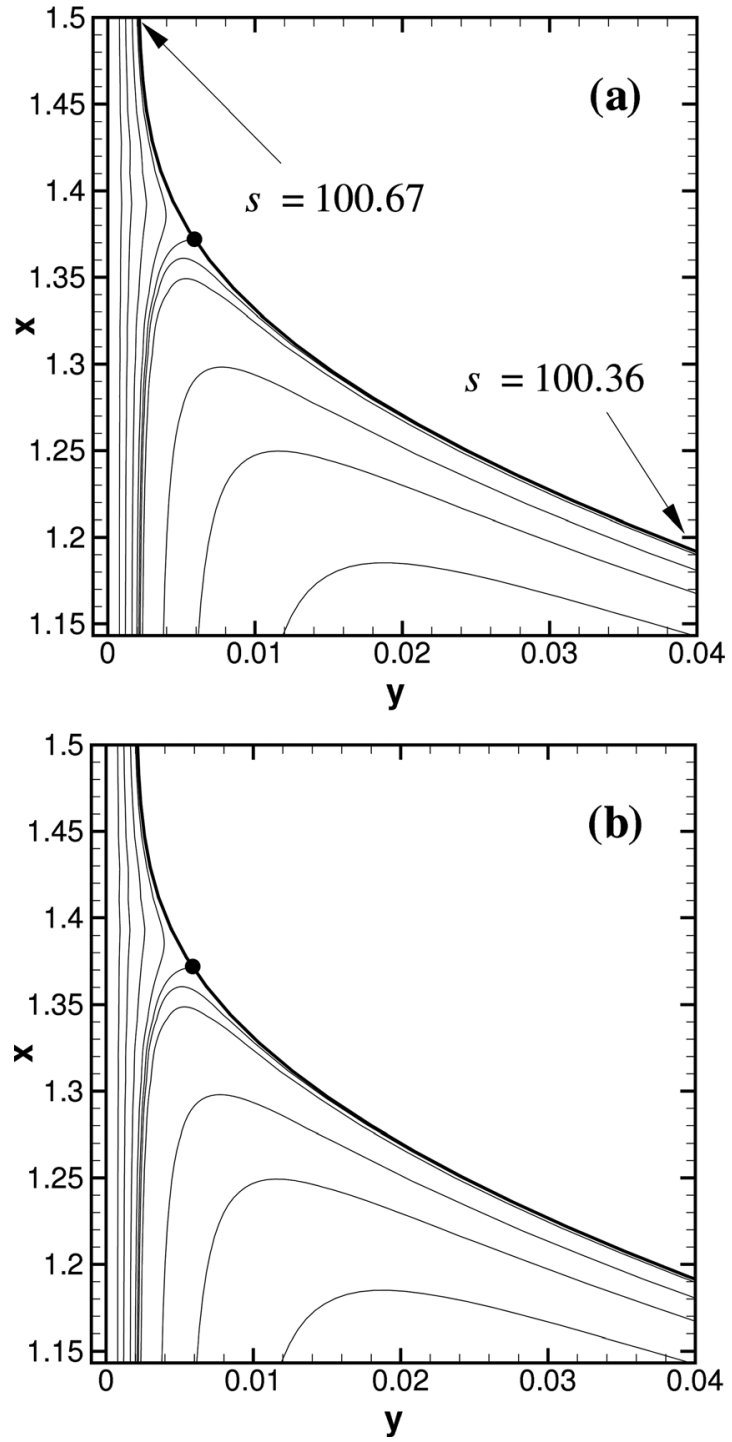

FIG. 12. Selected streamlines near the solid wall and the interface for $\beta=0.001$ and $C a=10^{-4}$ (a); $\beta=0$ and $C a=10^{-4}(\mathrm{~b})$.

conjectures; although in order to confirm them, more exhaustive numerical tests, including the use of a much more refined mesh when $\beta$ is equal to 0.01 and 0.1 , are required. For the particular value of $\beta$ selected, differences between the film thicknesses numerically obtained and those evaluated with the Landau-Levich law are smaller than $1 \%$ for $C a \geq 2.5 \times 10^{-5}$.

Figure 11 shows for $C a=10^{-4}$ and $\beta=0.001$ that the contaminated system is clearly behaving like a pure liquid; the related streamlines drawn in Fig. 12(a) reveal the presence of a stagnation point at the interface, as it is expected. Moreover, if those lines are compared with the streamlines depicted in Fig. 12(b) for the corresponding clean system, it is easy to conclude that they are almost identical.

The sequence of flow patterns presented in Fig. 10, in which the stagnation point is always in the bulk, evidences that the interfacial velocity does not change sign. Moreover, when $C a=0.009$ (Fig. 10(d)), the liquid is almost stagnant near the interface between $s=0$ and the bulk stagnation point marked with a black dot, as shown in Fig. 8(a). On the other hand, in a system free of surfactant, the interfacial ve-
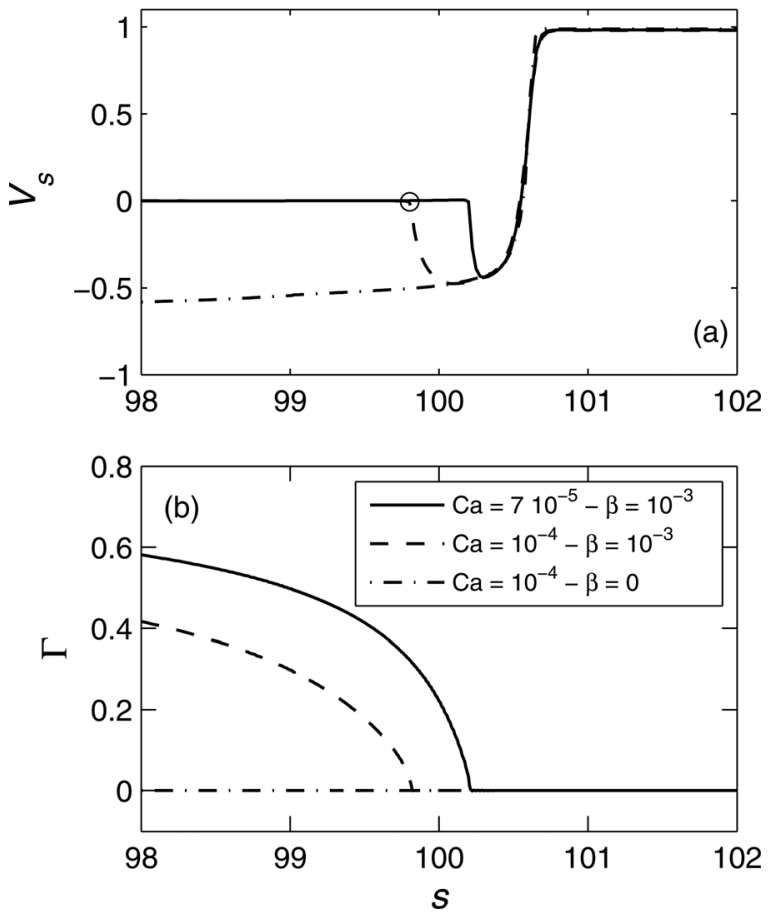

FIG. 13. Interfacial velocity (a) and distribution of surfactant along the free surface (b) for $C a=10^{-4}$.

locity increases toward both ends of the free surface, i.e., in the coordinate system adopted in this work it is negative from $s=0$ up to the stagnation point and positive from that point up to $s=s_{f}$. To underline those differences, in Fig. 13, we illustrate the free surface velocity (a) and the distribution of surfactant (b) for $C a=1 \times 10^{-4}$ and $\beta=0.001$ and 0 . It is interesting to note the two stagnation points that are present at the interface of the contaminated system: one located approximately at the same place as in the clean the system (it is marked with a black dot in Fig. 12(b), while the other one is placed close to it toward smaller values of $s$. The analysis of the free surface velocity for increasing $\mathrm{Ca}$ (not shown here) indicates that for $C a=2.5 \times 10^{-5}$, the region where that variable is negative is extremely small, but it increases with $\mathrm{Ca}$ becoming - as Fig. 13 shows-about 0.8 of the capillary length for $C a=10^{-4}$.

\section{B. The influence of inertia}

As we discuss at the beginning of this section, the parameter $m$ which measures the ratio between inertia and surface forces is equal to 52500 when the physical quantities take the values assigned by Park; thus, the Reynolds number is of order 1 or larger than 1 for $C a>10^{-4}$.

To analyze the influence of that parameter on the process under study, we carried out computations for $m=52500$ and for $\beta=0.01$ and 0.1 , within the range of $\mathrm{Ca}$ previously analyzed; the resulting predictions of the film thickness are reported in Fig. 14 where values for $R e=0$ are also drawn for comparison.

Naturally, the upper (dotted line) and lower (dashed line) curves of the film thickness are independent of $m$ since they depict the bounds: $4^{2 / 3}$ times Landau-Levich law and Landau-Levich law, respectively. However, the transition 


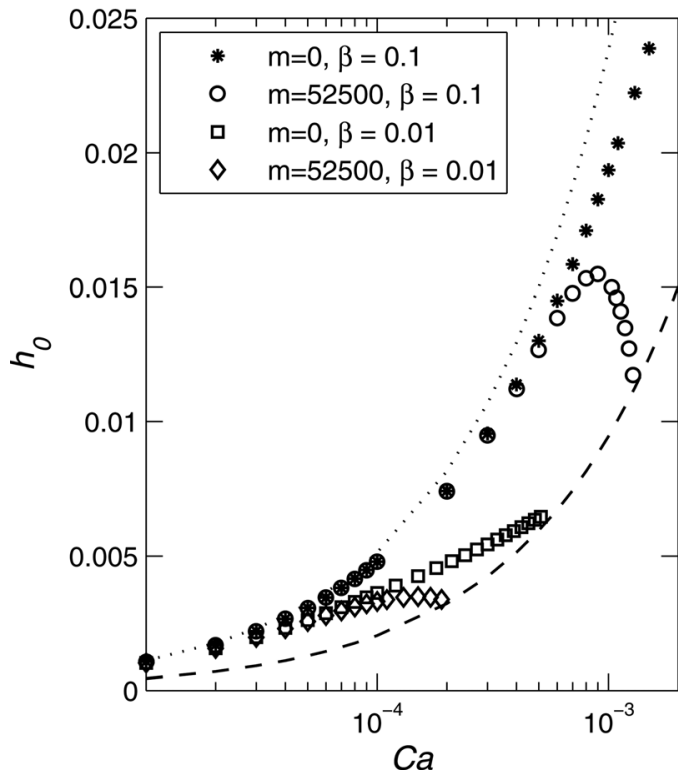

FIG. 14. Dimensionless film thickness vs. $C a$ for $m=0$ and 52500 and $\beta=0.01$ and 0.1 .

between those limits takes place in a narrower range of $\mathrm{Ca}$ when $m$ is augmented. Also, this transition is smoother for smaller $\beta$. Another interesting feature of the results depicted in that figure is that the contaminated system is more sensitive to the influence of inertia forces than the corresponding clean system. In fact, the upper triangles in Fig. 3 show that inertia $(m=52500)$ makes a clean system to deviate from the Landau-Levich law when $\mathrm{Ca}$ is about $2.2 \times 10^{-3}$, while in Fig. 14 we observe that inertia becomes noticeable at $C a=5 \times 10^{-5}$ when $\beta=0.01$ and at $C a=10^{-4}$ when $\beta=0.1$.

The thickness of the coated layer results from the interplay of viscous, inertia, and surface forces. At low coating speeds, the film thickness is determined by the competition between viscous and surface forces; however, as this variable becomes larger, $h_{0}$ will depend more and more on the ratio of inertia to surface forces, i.e., on the product $R e \times C a$. Since this ratio increases with $U^{2}$ inertia forces will counterbalance surface forces at lower values of $C a$ and the LandauLevich law will be approached faster.

To underline that concept, in Figure 15, we illustrate selected interfacial variables for $C a=0.0127$ and $\beta=0.1$ when inertia is disregarded $(R e=0)$ and when the Reynolds number is $635(R e=C a \times m=0.0127 \times 52500)$.

We observe that the velocity of the free surface along the static meniscus is very small and the transition between this velocity and the film velocity is very sharp when inertia forces are taken into account (dashed line in Fig. 15(a)).

We have already mentioned that the interfacial concentration of surfactant is ruled by surface convection; therefore, this variable must be closely related to the velocity of the free surface. Results reported in Fig. 15(b) show that, even though the difference between the maximum and minimum concentrations is larger when inertia force is computed, its distribution is smoother due to the very low velocities detected along the static meniscus. As a consequence, the surface traction presents a higher maximum
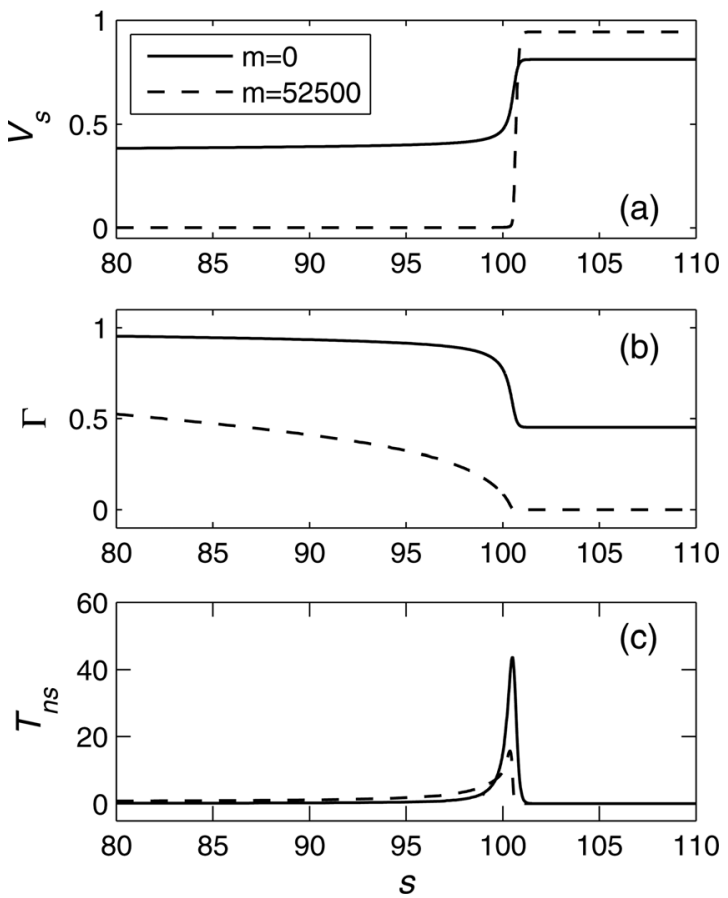

FIG. 15. Free surface velocities (a), interfacial concentrations of surfactant (b), and Marangoni tractions (c) along the free surface near the dynamic meniscus for $C a=0.00127$ and $\beta=0.1$; solid lines are for $m=0$ and dashed lines are for $m=52500$.

when the Reynolds number is set equal to zero (see Fig. 15(c)), and thus the effect of the surfactant on the film thickness will be overestimated if inertia is neglected in the calculations.

In a system free of surfactant, a thicker film is formed whenever the solid substrate is withdrawn faster; curves reported in Fig. 14 show that this is not the case when there is a surfactant adsorbed at the interface. Both for $\beta=0.01$ and $\beta=0.1$, the film thickness first increases as the substrate speed is augmented and then diminishes until the LandauLevich law is attained. The relative difference between the ticker film and the thickness computed when the LandauLevich law is reached is approximately equal to $7 \%$ and $28 \%$ when $\beta$ is equal to 0.01 and 0.1 , respectively. Numerical solutions not presented here show that this difference is an increasing function of the elasticity number when $\beta$ is within the range $0.1-0.2$.

\section{CONCLUSION}

The goal of this work is the numerical analysis of the dip coating process when a totally insoluble surfactant is present; for that purpose, we made slight changes to an already validated numerical code previously employed to study the same process with soluble surfactants. ${ }^{8}$ Our predictions about the film thickness deposited compare well with those obtained by Park ${ }^{9}$ who used a simpler one-dimensional model based on the method of matched asymptotic expansion; this model does not account for inertial effects.

The difference between the film thicknesses predicted by both models increases as the capillary number reaches a value large enough so that viscous forces overtake the elastic ones. Logically, at the point viscous forces wipe out elastic 
effects, the thickness ratio becomes one and the Landau-Levich law is recovered. This transition occurs at higher $C a$ values as the strength of the surfactant increases, i.e., as $\beta$ increases.

Inertial effects become important in the dip coating process when liquids of low viscosities are employed. In such a case, capillary values as low as $10^{-3}$ might imply $R e$ values as large as 100. Our predictions considering the $R e$ values arising from the physical properties adopted by Park show that the inertial forces considerably affect the coating thickness. In fact, for $\beta=0.1$, the values of $C a$ at which the Landau-Levich law is recovered are approximately $9.0 \times 10^{-3}$ and $1.4 \times 10^{-3}$ when $R e$ is zero and 73.5, respectively.

The results presented in this work complete the picture about the flow pattern appearing in the dip coating process of a plane substrate. In a previous work, ${ }^{8}$ we first showed the streamlines when an inert soluble solute $(\beta=0)$ is employed, which are identical to the streamlines produced by a pure liquid; this flow pattern is characterized by the presence of a single stagnation point located at the gas-liquid interface where the film thickness is approximately three times the final film thickness $\left(h_{0}\right)$. Then, as we increased the value of $\beta$, we observed that the stagnation point located at the interface moves away from the film region-i.e., toward smaller values of the arc length-while a second stagnation point located closer to the film region appears in the bulk. This second stagnation point is a saddle point which presents a closed swirl and two liquid streams, one of them is directly dragged by the solid toward the film region, while the second one turns around the swirl joining the gas-liquid interface and moving along the free surface. Both streams joint just at the saddle point and move together carrying the liquid that will be deposited on the substrate.

More recently, we studied how the flow pattern just described changes as the surfactant becomes less soluble. For that purpose, we continuously reduce the adsorption velocity of the surfactant, which is measured through the Stanton number, until it becomes zero. As the Stanton number is reduced, the close swirl originated at the saddle point grows larger and the stagnation point located at the free surface moves further and further away from the film. Eventually, the surfactant becomes completely insoluble; at that stage, the stagnation point at the interface is not longer there, the liquid velocity is positive all along the interface and the stagnation point in the bulk very much resembles the split ejection streamline pattern observed by Díaz and Cerro ${ }^{14}$ in their study about the transition from Langmuir-Blodgett to dip coating flow.

Finally, in the present work, we have described how the flow pattern produced by a totally insoluble surfactant evolves as the coating speed is augmented, and the classical result of Landau and Levich is recovered.

${ }^{1}$ L. Landau and B. Levich, "Dragging of a liquid by a moving plate," Acta Physicochim. URSS 17, 42 (1942).

${ }^{2}$ D. A. White and J. A. Tallmadge, "Theory of drag out of liquids on flat plate," Chem. Eng. Sci. 20, 33 (1965).

${ }^{3}$ S. D. R. Wilson, "The drag-out problem in film coating theory," J. Eng. Math. 16, 209 (1982).

${ }^{4}$ A. de Ryck and D. Quér,é, "Gravity and inertia effects in plate coating," J. Colloid Interface Sci. 203, 278 (1998).

${ }^{5} \mathrm{O}$. Ou Ramdane and D. Quéré, "Thickening factor in Marangoni coating," Langmuir 13, 2911 (1977).

${ }^{6}$ A. Q. Shen, B. Gleason, G. H. McKinley, and H. A. Stone, "Fiber coating with surfactants," Phys. Fluids 14, 4055 (2002).

${ }^{7}$ R. Krechetnikov and G. M. Homsy, "Experimental study of substrate roughness and surfactant effects on the Landau-Levich law," Phys. Fluids 17, 102108 (2005).

${ }^{8}$ D. M. Campana, S. Ubal, M. D. Giavedoni, and F. A. Saita, "Numerical prediction of the film thickening due to surfactants in the Landau-Levich problem," Phys. Fluids 22, 032103 (2010).

${ }^{9}$ C.-W. Park, "Effects of insoluble surfactants on dip coating," J. Colloid Interface Sci. 146, 382 (1991).

${ }^{10}$ J. Ratulowski and H.-C. Chang, "Marangoni effects of trace of impurities on the motion of long bubbles in capillaries," J. Fluid Mech. 210, 303 (1990).

${ }^{11}$ R. Krechetnikov and G. M. Homsy, "Surfactant effects in the Landau-Levich problem," J. Fluid Mech. 559, 429 (2006).

${ }^{12}$ P. Groenveld, "Low capillary number withdrawal," Chem. Eng. Sci. 25, 1259 (1970)

${ }^{13}$ H. A. Stone, "A simple derivation of the time-dependent convective-diffusive transport equation for surfactant along deforming interfaces," Phys. Fluids A 2, 111 (1990).

${ }^{14}$ M. E. Diaz and R. L. Cerro, "Transition from split streamlines to dip-coating during Langmuir-Blodgett film deposition," Thin Solid Films 460, 274 (2004). 